\title{
MicroRNAs: New Biomarkers for the progression of Coronary Artery Diseases
}

\author{
Yaxi Chen ${ }^{1}$ \\ ${ }^{1}$ Dpartment of Biomedical Engineering, Beihang University, China
}

\begin{abstract}
Cardiovascular diseases (CVDs), especially the coronary arterial diseases (CADs), have become the main cause of death all around the world, attracting attentions from the whole society. Even though considerable progresses have been made for the treatment of CADs, many clinical challenges remain to be overcome. In particular, effective biomarkers for CADs need to be developed to facilitate the early diagnosis and thus early treatment of the disease. Recently, the dysregulation of microRNAs (miRNAs) has been found to be involved in the progression of multiple CADs, manifested as altered levels of miRNAs at different disease phases, suggesting that miRNAs may be capable of serving as promising biomarkers for CADs. Here, we attempt to evaluate the possibility of miRNAs as biomarkers for CADs and compare these markers with previously reported ones. In this review, we will summarize the basic concepts and advances for CADs and miRNAs, with a special emphasis on miRNAs in the progression of CADs.
\end{abstract}

\section{Introduction}

Cardiovascular diseases (CVDs), as the most common heart disease, have been seriously threatening human lives. Among the heart diseases, coronary artery disease (CAD) is one of the main reason of death all over the world ${ }^{[1]}$. CAD often refers to a serious condition caused by the buildup of plaques in the coronary arteries, which makes the vessels become stiff and narrow to impede the flow of oxygen-rich blood to the heart, and as a result, a heart attack or hemorrhagic stroke will be caused ${ }^{[2]}$. The development of CAD is constant and unpredictable, which can cause major adverse cardiovascular events, such as myocardial infarction (MI), angina and cardiovascular death $^{[2]}$. By now, percutaneous coronary intervention (PCI) is the most common approach to treat $\mathrm{CAD}$, after which most patients have significantly reduced angina and improved quality of life ${ }^{[3]}$. However, despite the continuous improvement of the medical equipment and technology, as time goes by, the long-term results of stent implantation are still affected by the risk of in-stent restenosis (ISR). At present, $\operatorname{ISR}^{[4][4]}$, MI, stent thrombosis and several complications after PCI remain to be challenging problems ${ }^{[5]}$, which have gradually attracted widespread attention. As a result, identification of timely and reliable diagnostic- and prognostic- biomarkers for CADs is of great value for the precaution and medical treatment of the disease ${ }^{[6],}{ }^{[7]}$. Recently, increasing evidence has suggested that multiple microRNAs (miRNAs) are dysregulated in CADs. Considering the existence of miRNAs in peripheral blood mononuclear cells as well as biofluids such as serum, urine, cerebrospinal fluid, they may be capable of serving as promising non-invasive biomarkers for detecting CADs and monitoring the disease progression.

Here, we will review the state of miRNAs as biomarkers for CADs, including the advantages and disadvantages of current approaches, and underline their limitations but also the huge potential for further clinical applications.

\section{Basic biology and mechanisms of miRNAs in CADs}

\subsection{Basis biology of miRNAs}

miRNA was initially discovered from C elegans in 1993 by Victor Ambros' laboratory while studying the gene lin$14^{[8]}$, which refers to a class of endogenous, conserved, single-stranded, regulatory small non-coding RNAs of about $22 \mathrm{nt}$ in length. Generally, miRNAs are partially complementary to one or more messenger RNAs (mRNAs) ${ }^{[8]}$, as a result, miRNAs can modulate gene expression at the post-transcriptional level by translational repression, mRNA cleavage and deadenylation and so on ${ }^{[8]}$. As for the production of miRNAs, they are often synthesized from the primary miRNAs (pri-miRNA), which are firstly transcribed in the nucleus and then processed into premiRNAs by Drosha. Afterward, pre-miRNAs are exported to the cytoplasm and eventually processed by Dicer complex, resulting in mature miRNAs ${ }^{[9]}$ (Figure 1). Furthermore, miRNAs are involved in the regulation of various biological processes and play important roles in gene expression, cell differentiation, cell proliferation and migration, apoptosis and stress response ${ }^{[10]}$.

In addition, miRNAs are characterized with tissue-

\footnotetext{
* Corresponding author: chen17375470@buaa.edu.cn
} 
specific expressions, which provides an opportunity to determine the normal- or disease- state of a specific tissue ${ }^{[11]}$. Additionally, circulating miRNAs are existent in body fluids, especially in blood, plasma, serum, and even saliva, which equip miRNAs with convenient availability to act as potential biomarkers for multiple diseases. Currently, a plenty of researches have shown that the dysfunction of miRNAs is implicated in a growing number of CVDs, including CAD, MI, heart failure, hyper tension and stroke ${ }^{[12-14]}$. For example, in the patients with CADs, several miRNAs such as miR-126-3p, miR-21 render significantly increased levels while some other miRNAs such as miR-92a, miR-126, miR-17 show reduced levels ${ }^{[15]}$. On above basis, detection of related miRNAs may help us to detect multiple CVD

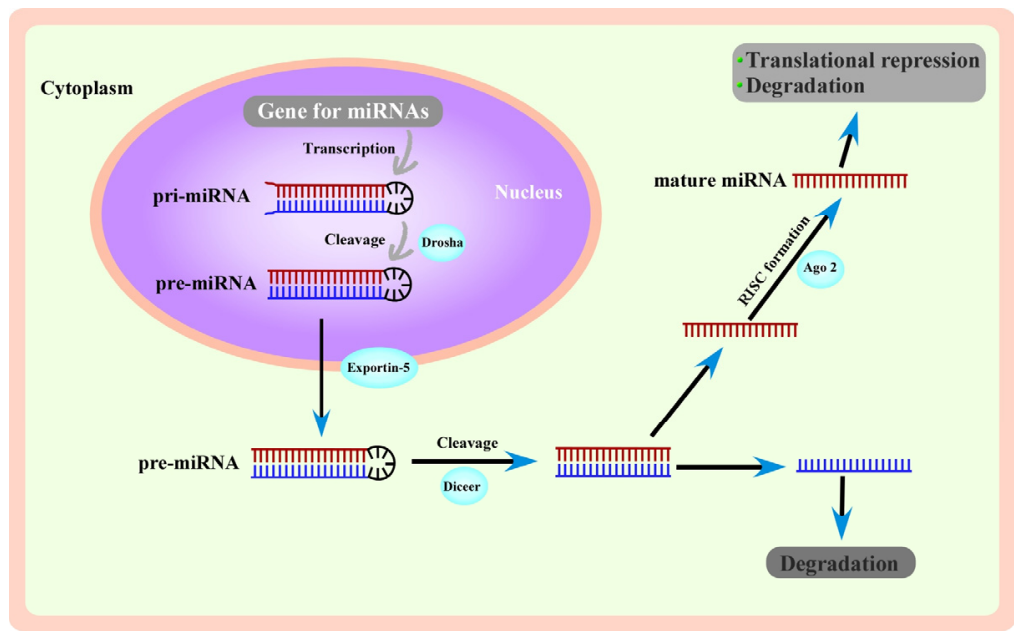

Figure 1 A schematic representation of the miRNA biogenesis. Firstly, pri-miRNAs equipped with 5'-caps and 3'-polyA tails are generated from the transcription in the nucleus. Drosha then cleaves and removes the caps and tails, resulting in the pre-miRNAs. Next, pre-miRNAs are exported to the cytoplasm via Exportin-5. Subsequently, in the cytoplasm, the pre-miRNAs are cleaved by Dicer, generating two single-strand RNA, among which one single-strand RNA is degraded, the other one is further processed into mature miRNA via RISC complex. And the mature miRNAs will either perform translational regulation effects or commit degradation.

\subsection{Mechanisms of miRNAs in CVDs}

Considerable studies have reported that miRNAs can play important regulation roles in a variety of physiological processes within the cardiovascular system, and the dysregulation of miRNAs could lead to severe consequences. Moreover, miRNAs can perform the modulation role in CVDs by affecting the functions of several critical cells, including vascular smooth muscle cells (VSMCs) and vascular endothelial cells.

Firstly, miRNAs are related to VSMCs. In the cardiovascular system, contact inhibition of normal cardiovascular cells is necessary to keep the normal size of heart and vessels, wherein VSMCs are the main elements of the middle layer of the artery, playing an important role in maintaining the physiological function of normal arteries ${ }^{[14]}$. The lack of contact inhibition of VSMCs in arterial walls could lead to the overproliferation of VSMCs as well as their extracellular matrix, thus resulting in vascular neointimal formation and arterial stenosis, which are typical pathological changes in atherosclerosis, CADs, and stroke ${ }^{[14]}$. Above processes are closely associated with miRNAs, for instance, Sun et al ${ }^{[14]}$ reported that miR-145 had critical influence on the contact inhibition of VSMCs, which would affect the cell proliferation and migration, thereby leading to restenosis ${ }^{[16,17]}$. Besides, the development of plaques may be directly caused by miR-221 and miR-222 through stimulating VSMCs proliferation and accelerating neointima formation ${ }^{[18]}$.
In addition, miRNAs are related to vascular endothelial cells as well. Generally, vascular endothelial cells have a barrier function within the vascular structure, which can maintain the integrity and permeability of blood vessels, thereby exerting important effects on vascular physiology such as related immune response and inflammation ${ }^{[19]}$. Some miRNAs are able to influence the angiogenesis by modulating the angiogenic mediators like vascular endothelial cell growth factor (VEGF) $)^{[20]}$. Additionally, miRNAs could also modulate the function of endothelial cells and the formation of arterial thrombus through NF- $\kappa B$ which is critical for the thrombogenic thrombin in generating procoagulant responses to amplify inflammation $^{[21]}$. For example, miR-181b can possess anti-inflammatory actions and inhibit downstream NF- $\kappa B$ signaling in response to TNF- $\alpha$, it can also directly inhibit upstream NF- $\kappa$ B signaling induced by thrombin ${ }^{[21]}$.

Of course, apart from above mechanisms of miRNAs in performing modulatory roles in CADs, miRNAs could also regulate the translation of a target mRNA, protein synthesis, cell cycles and so forth ${ }^{[22,23]}$. Therefore, miRNAs can exert important regulatory roles in CAD related processes, thus contributing to the initiation or progression of CADs.

\section{3 miRNAs serve as biomarkers for the progression of CADs}

In terms of the cardiovascular diseases, several molecules have already been used as biomarkers, such as the low- 
density lipoprotein cholesterol, circulating brain natriuretic peptide (BNP) and so on ${ }^{[24]}$. Unfortunately, few of these biomarkers have been currently transformed into practical diagnostic applications $\left.{ }^{[25,} 26\right]$. With the development of precision medicine and next generation sequencing, miRNAs have shown great advantages in serving as diagnostic and prognostic biomarkers for CADs due to its high specificity, sensitivity and convenient availability $^{[27]}$. Here, we will review the potential of miRNAs in acting as biomarkers for the progression of CADs, exemplified from the early stage of atherosclerosis to acute myocardial infarction, and then to the late stage of heart failure. Finally, the advances of miRNAs in the prognosis of PCI will also be discussed.

\section{1 miRNAs in atherosclerosis (As)}

Atherosclerosis (As) often refers to the hardening and narrowing of the arteries, and neointimal formation is one of the main pathological changes of atherosclerosis, which include variation of endothelial cell physiology and hyperplasia of $\mathrm{VSMCs}^{[28]}$. These changes will cause a gradual narrowing of the vessel's lumen, leading to thrombus formation and vessel occlusion ${ }^{[28]}$. At present, As is the typical origin of cardiovascular diseases such as heart attacks, strokes and peripheral vascular disease and so on ${ }^{[29]}$. Therefore, identification of biomarkers for As can facilitate the early diagnosis of multiple cardiovascular diseases. As is often accompanied by inflammation, while monocytes and macrophage cells play the dominant roles in inflammatory responses ${ }^{[30]}$. Hence, several inflammatory factors have been suggested as potential biomarkers for As, including microparticles (MPs), oxidized LDL (oxLDL) and so forth ${ }^{[31]}$ (Table 1). However, the unclear mechanisms, low sensitivity, low specificity, as well as poor repeatability restrict their wide clinical applications

Recently, multiple studies have reported that miRNAs are dysregulated during the development of As (Table 2), for example, several miRNAs such as miR-155, miR-33 are upregulated in $\mathrm{As}^{[30,32]}$. Considering the function of miRNAs in macrophage polarization, they may be able to serve as important regulators and predictors for As. Among the multiple dysregulated miRNAs in As, miR155 and miR-33 have also been thought as the potential targets to decelerate the development of As. miR-155 is upregulated in response to pro-inflammatory stimulants, and its repression may be a viable strategy to reduce the inflammatory response ${ }^{[32]}$. Li et al ${ }^{[33]}$ suggested that the increased level of miR-155 could relieve inflammation and foam cell formation via the signaling pathway of miR155-CARHSP1-TNF- $\alpha$, but it was also claimed that the anti-inflammatory function of HDL is conferred through miRNA-33. Besides, miR-33 inhibition in macrophages has been verified to be able to enhance cholesterol efflux and reduce As lesions in mice ${ }^{[32]}$. Additionally, miR-21, a pro-survival gene for VSMCs, is another important miRNA in As, which has been demonstrated to be capable of impacting neointimal formation ${ }^{[28]}$. Additionally, the in vitro studies on miR-21 have shown that it is an essential regulator of VSMC proliferation, which can inhibit cell apoptosis $^{[28]}$. Furthermore, it was revealed that lack of miR-21 attenuated neointima formation and macrophage activation, resulting in a less inflammatory phenotype ${ }^{[34]}$.

By now, miRNAs have rendered great potential for clinical applications, but more mechanistic studies remain to be performed to gain deeper understanding on the modulatory effects of miRNAs in As. Additionally, by combining above alterations of miRNAs, it is promising to identify effective diagnostic hallmarks for As, so as to provide valuable clinical information for early treatment of As.

Table 1. Advantages of miRNAs biomarkers over the protein ones for AMI, As, HF and ISR

\begin{tabular}{|c|c|c|c|}
\hline Diseases & Protein biomarker & miRNA biomarkers & $\begin{array}{c}\text { Advantages of miRNA } \\
\text { biomarkers }\end{array}$ \\
\hline AMI & $\begin{array}{l}\text { CK-MB }{ }^{* 25]} \\
\text { Myoglobin (MYO) } \\
\text { Troponin C }\end{array}$ & $\begin{array}{l}\operatorname{miR}-519 e-5 p^{[36]} \\
\operatorname{miR}-199 a-1^{[37]} \\
\operatorname{miR}-26 a-1^{[37]} \\
\operatorname{miR}-146 a^{[37]}\end{array}$ & $\begin{array}{l}\text { Large choice space due to the } \\
\text { various miRNAs able to server } \\
\text { as biomarkers. }\end{array}$ \\
\hline As & $\begin{array}{l}\text { Pentraxin } 3(\text { PTX-3 })^{[35]} \\
\text { LOX-1*31] } \\
\text { RANTES }^{*}[31]\end{array}$ & $\begin{array}{l}\operatorname{miR}-33^{[32,38]} \\
\operatorname{miR}-155^{[32]}\end{array}$ & $\begin{array}{l}\text { Convenient availability: } \\
\text { blood, saliva, and urine } \text { et al. }\end{array}$ \\
\hline HF & Natriuretic peptide ${ }^{[39]}$ & $\begin{array}{l}\operatorname{miR}-1^{[40]} \\
\operatorname{miR}-21^{[41]} \\
\operatorname{miR}-132^{[42]}\end{array}$ & High specificity. \\
\hline ISR & $\begin{array}{l}\text { CD } 45 \text { p platelet count }{ }^{[43]} \\
\text { C-reactive protein }{ }^{[44]}\end{array}$ & $\begin{array}{l}\operatorname{miR}-143^{[45]} \\
\operatorname{miR}-93-5 \mathrm{p}^{[46]}\end{array}$ & High stability in body fluids. \\
\hline
\end{tabular}

CK-MB*: Creatine kinase-MB; LOX-1* : Lectin-like oxidized LDL receptor-1; RANTES*': Regulated on activation, normal T cellexpressed and secreted

Table 2. miRNAs associated with As, AMI, HF and ISR

\begin{tabular}{ccclc}
\hline Diseases & miRNAs & Expression & Potential action & $\begin{array}{c}\text { Refer } \\
\text { ence }\end{array}$ \\
\hline \multirow{3}{*}{ As } & miR-155 & $\uparrow$ & Relieve inflammation and foam cell formation & {$[32]$} \\
& miR-33 & $\uparrow$ & Reduce cholesterol efflux and increase Atherosclerotic lesions & {$[31]$} \\
& miR-21 & $\downarrow$ & Impact neointimal formation/ Inhibit cell apoptosis & {$[28,34]$} \\
AMI & miR-30a & $\uparrow$ & Regulation of cardiovascular function after AMI & {$[47]$}
\end{tabular}




\begin{tabular}{|c|c|c|c|c|}
\hline & miR-133 & $\uparrow$ & Promote arrhythmogenesis & {$[48]$} \\
\hline & miR-1 & $\uparrow$ & Promote arrhythmogenesis & [48] \\
\hline & $\operatorname{miR}-27 \mathrm{a}$ & $\downarrow$ & Stimulate left ventricular contractility & [48] \\
\hline \multirow[b]{2}{*}{ HF } & $\operatorname{miR}-21$ & $\uparrow$ & $\begin{array}{l}\text { Cause profibrotic effect and change cardiomyocyte } \\
\text { morphology }\end{array}$ & [41] \\
\hline & miR-195 & $\uparrow$ & $\begin{array}{l}\text { Induce cardiac growth with disarrangement of } \\
\text { cardiomyocytes; ventricular expansion with degeneration in } \\
\text { cardiac function }\end{array}$ & [49] \\
\hline \multirow{2}{*}{ ISR } & miR-21 & $\downarrow$ & $\begin{array}{l}\text { Weak neointima formation and macrophage activation; lead to } \\
\text { a less inflammatory phenotype }\end{array}$ & [34] \\
\hline & miR-143 & $\downarrow$ & $\begin{array}{l}\text { Lead to a less inflammatory phenotype and modulate VSMC } \\
\text { phenotypes }\end{array}$ & [45] \\
\hline
\end{tabular}

\section{2 miRNAs in acute myocardial infarction (AMI)}

With the development of As, multiple CVDs would be caused, including myocardial infarction (MI), angina, arrhythmia and so on ${ }^{[50]}$. Among these, MI is the typical disease caused by As, which is defined as myocardial cell death caused by prolonged ischemia. MI is a kind of typical cardiovascular disease, and it is considered the key target for the treatment of the patients with $\mathrm{CAD}^{[51]}$. And acute myocardial infarction (AMI) is a cardiovascular disease with rather high morbidity, so accurate and reliable diagnosis of AMI is earnestly needed to promote the assessment of the risks for the patients so that they can undergo timely reperfusion therapy and thus restore blood flow $^{[51]}$.

Currently, creatine kinase-MB (CK-MB) isoenzymes and cardiac troponins are recognized as the mainstream biomarkers, even as the "gold standard", for the detection of $\mathrm{AMI}^{[25]}$ (Table 1). Although these markers have been proved to have important clinical value, there are some limitations. For instance, despite that C-reactive protein (CRP) showed increased level in unstable angina patients, it could not be used as a diagnostic marker due to the low sensitivity and specificity ${ }^{[35]}$. Additionally, these biomarkers lack satisfying availability because their large sizes make them difficult to enter or diffuse to body fluids such as urine and blood, which hinders their practical applications in clinics.

Different from the large-size biomarkers, miRNAs were recently proposed to be novel biomarker candidates for the diagnosis of AMI. Previous studies suggested that miRNAs could be released from the heart into circulation system upon myocardial injury ${ }^{[23,52]}$ (Table 2). Under disease conditions, the levels of several miRNAs such as miR-208, miR-499, miR-1, miR-133 would change ${ }^{[53]}$. Among these, upregulation of miR-1, a heart and muscleenriched miRNA, has been previously reported in postinfarction patients to act as a surrogate parameter of acute cardiac damage ${ }^{[54]}$. Moreover, it was shown that the level of plasma miR-1 was elevated in the patients with AMI compared to the control group ${ }^{[54]}$. In particular, the content of miR-1 in serum peaked at day 9 after infarction and then decreased to lower but still elevated level at 6-month follow-up ${ }^{[54]}$. Additionally, increasing studies have shown that circulating muscle-derived miRNAs such as miR-1, miR-208 and miR-499 could leak out from the necrotic myocardium and release into the circulation system during the early stage of AMI, thus enabling them to serve as promising biomarkers for $\mathrm{AMI}^{[55]}$. Moreover, Wang et al ${ }^{[56]}$ also demonstrated that the levels of miR-1, miR-208 and miR-499 increased in plasma. Besides, the levels of miR208 and miR-499 could rapidly decrease after PCI, suggesting that miR-208 and miR-499 have the potential to be biomarkers for AMI prognosis ${ }^{[56]}$.

In terms of specificity, miRNAs are often involved in various processes in the infarcted heart, including neovascularization, progenitor-cell-mediated repair, cardiomyocyte cell death and proliferation ${ }^{[57]}$. Wang et $a l^{[36]}$ has evaluated the ability of miR-519e-5p in distinguishing AMI from ischemic stroke (IS) and pulmonary embolism (PE), which showed that miR-519e$5 \mathrm{p}$ had a low level in AMI patients but opposite in the other ischemic diseases, thereby proving that miR-519e-5p could also be used as a potential biomarker for $\mathrm{AMI}^{[36]}$. On the contrary, the increased miR-21-5p and miR-361-5p not only exist in AMI, but also in PE and $\mathrm{IS}^{[37]}$. In addition, the levels of miRNAs are also associated with the degrees of AMI. Xue et $a l^{[37]}$ investigated the different serum levels of miRNAs in the patients with different degrees of AMI, which found that the serum miRNAs such as miR26a-1, miR-146a and miR-199a-1 could elevate with the increase of the number of coronary lesions. Under the condition of hypoxia, miR-186, miR-210, and miR-150 in myocardial ischemia had been previously reported to be upregulated $^{[58]}$. Moreover, a study showed close relationship between declined expressions of miR-19a as well as miR-19b and age-related heart remodeling, supporting that miRNAs can indeed impact the development of cardiac aging and heart failure ${ }^{[59]}$.

\section{3 miRNAs in heart failure (HF)}

Heart failure (HF) is a debilitating epidemic disease influencing approximately $1 \% \sim 2 \%$ of adult population in the modern society. HF means that the heart is not pumping as well as it should be, which is the final manifestation of almost all types of CVDs, including CADs, heart attack, cardiomyopathy and so forth ${ }^{[60]}$. Generally speaking, the failing heart is always accompanied by cardiomyocyte death and fibrosis ${ }^{[41]}$.

In recent years, abnormal expressions, both upregulation and down-regulation, of numerous miRNAs have been proved to be related to HF, including miR-21, miR-132, miR-210 and so on ${ }^{[15]}$ (Table 2). miR-21 is upregulated in the patients with symptomatic HF, which has been related to myocardial disease because of the activation of mitogen-activated protein kinase (MAPK) 
signaling in fibroblasts and the induction of cardiac fibrosis, thus leading to a profibrotic effect and change cardiomyocyte morphology ${ }^{[41]}$. Masson et a ${ }^{[42]}$ claimed that the level of miR-132 could increase with the severity of HF, however, another study reported that the lower level of miR-132 could improve the risk of prediction for HF readmission, so the controversy on miR-132 still needs to be further clarified. In addition, circulating miR-1306 was positively related to the adverse clinical outcome of acute HF patients, and elevated levels of miR-499 were also strongly associated with the increased risk of HF or related death $^{[61]}$. Additionally, miR-195 has been suggested to be able to induce cardiac growth with disarrangement of cardiomyocytes and finally ventricular expansion with degeneration in cardiac function ${ }^{[49]}$. Furthermore, miR195 is also verified to be able to target several genes to participate in several signaling pathways, such as Gadd45g, Map2k1 and Raf1 genes, which are associated with the MAPK signaling pathway ${ }^{[49]}$. Additionally, some miRNAs such as miR-22, miR-221 and miR-423 rendered decreased levels in the patients with improved $\mathrm{HF}^{[41]}$.

Based on above advances, it is promising to combine the observations of several miRNAs to develop diagnostic biomarkers for HF, so as to facilitate the monitoring and even the treatment of HF. Further studies remain to be performed to validate and evaluate the effects of miRNAs in HF.

\section{4 miRNAs and in-stent restenosis (ISR)}

At present, percutaneous coronary intervention (PCI), also known coronary angioplasty, is a major clinical approach for treating multiple $\mathrm{CADs}^{[5]}$. However, many clinical challenges about PCI still need to be overcome, in particular, in-stent restenosis (ISR) remains an important factor affecting the prognosis of PCI. Vascular responses to PCI include elastic recoil, dissection and neointimal formation ${ }^{[62]}$. The stents may promote neointimal hyperplasia, which is the main cause of ISR $^{[62]}$. Nowadays, although CT coronary angiography is a commonly used method for the diagnosis of ISR, the patients will receive significant radiation and contrast agents which often have adverse influence ${ }^{[46,}{ }^{63}$. Therefore, non-invasive biomarkers for ISR and modified stents with superior properties will be of great value in improving PCI efficacy.

Currently, several miRNAs have been found to be associated with ISR (Table 2). A remarkably decreased level of serum miRNA-143 has been reported in ISR group compared with the control one, showing its potential in serving as an effective biomarker ${ }^{[45]}$. It has been suggested that the major cause of ISR is the migration and proliferation of VSMCs, which could induce the generation of neointima and further lead to restenosis ${ }^{[6,45]}$. A study based on a mouse model of ISR demonstrated that the lack of miR-21 could weaken neointima formation and macrophage activation, thus resulting in a less inflammatory phenotype ${ }^{[34]}$. Furthermore, another study suggested that miR-21 inflection could enhance wound recovery and solve the inflammatory response, which could be used to improve the clinical effects of currently available stents and reduce the risks of restenosis and stent thrombosis ${ }^{[34]}$. Additionally, a miRNA nanoparticle-coated coronary stent was developed and then implanted into the coronary arteries of a rabbit model, which showed that proliferation of smooth muscle cells was inhibited and ISR was effectively prevented ${ }^{[64,}{ }^{65]}$. Besides, Izuhara et al ${ }^{[66]}$ developed a system that combined miR-126 nanoparticles with the stents, which can release miR-126 to the local area for about two weeks. Then the effectiveness of the miR-126 coated stent was evaluated in a rabbit model, which showed a significantly inhibition effect on the development of neointimal hyperplasia ${ }^{[66]}$. Therefore, miRNA interference strategy is prospective for improving the stents or preventing ISR by inhibiting the neointimal formation.

\section{Evaluation and challenges of miRNAs as diagnostic biomarkers}

From multiple perspectives, miRNAs have tremendous potential in serving as non-invasive biomarkers for CADs. However, current clinical applications of miRNA are still not extensive due to several pressing technical challenges.

By now, most researches of miRNA on diagnosis or prediction are still performed at animal level and lack preclinical studies, let alone the actual clinical ones, thereby restricting the clinical applications. Besides, the number of patients for the study of miRNA biomarkers is in short supply and the tracking time is still short, which cause the lack of sufficient evidence to confirm whether miRNAs are reliable biomarkers for the diagnosis, treatment and prognosis of $\mathrm{CADs}^{[15]}$. Additionally, it remains a great challenge to make a distinction of specific miRNA biomarkers from different stages of the disease ${ }^{[9]}$. For instance, the protective effect of miR-21-5p on cell apoptosis induced by ischemia has been proved both in vivo and in vitro, which validated that miR-21-5p indeed played a role in the early phase of AMI, but the dynamic expression of miR-21-5p during AMI is still unclear ${ }^{[67]}$. In addition, although there are many researches on miRNAs at present, the analysis of the same miRNA for the same disease from different studies rendered different, sometimes even the opposite results. One reason for the controversy may be that miRNAs can be generated via several mechanisms or involved in multiple signaling pathways under a certain disease condition, so the actual underlying mechanism for a disease is hardly to be elucidated due to these complications ${ }^{[9]}$. Hence, deeper investigations are needed to clarify the involvement of miRNAs in various diseases, so as to accelerate their applications to the clinic.

Another problem is that the recovery rate of miRNAs still needs to be improved. By now, the most common fluid source for miRNA quantification comes from blood, with other fluids still being under exploration. For example, saliva and urine could be very fascinating candidates for further investigation due to that saliva often contains high contents of circulating miRNAs and can provide a quite convenient way to collect the samples ${ }^{[68]}$. However, it remains a challenging work to effectively extract the small amount of nucleic acids from the biofluids due to their 
small sizes, high sequence homology across family members. In terms of miRNA extraction, the currently used procedures are often carried out with the extraction kits, and the process can be generally divided into three steps: lysis, organic extraction, and precipitation, among which precipitation is the most critical step that mainly determine the extraction efficiency ${ }^{[69]}$. As for miRNA detection, the traditional northern blot is regarded as the "gold standard", however, it is lowly-sensitive, timeconsuming, thereby requiring a large number of RNA samples. Currently, several alternative methods such as real-time PCR, microarray technology are also adopted for miRNA detection, but these techniques are so complex that they usually require professional laboratory skills. Besides, false positives may frequently be generated during the amplification process ${ }^{[70]}$. Although various detection methods such as qRT-PCR, northern blot as well as microarray are commonly used to detect miRNAs, their extensive applications are restricted by the existent limitations, including the long processing time, high requirements of sample volumes, frequently occurred false-positive results and so on. And in practical applications, several methods are often used together so as to promote the accuracy of the results, which make the detection process more complicated and increase the overall costs ${ }^{[70]}$. Therefore, more convenient, more reliable, faster and cheaper techniques are expected to be developed in the near future.

\section{Progression of CAD}

$\begin{gathered}\text { Atherosclerosis } \\ \text { (As) }\end{gathered}$
miR-29a
miR-126
miR-212

\section{Conclusion and future directions}

In recent years, CADs seriously threat human life and bring heavy burden to the society, and considerable progresses have been made to better understand the mechanisms responsible for CADs. Development of timely and reliable diagnosis approaches can enable more effective therapies for CADs and thus reduce mortality. Multiple studies have demonstrated that multiple miRNAs are closely associated with CADs, suggesting their roles as promising hallmarks as well as potential treatment targets for CADs. Here, we have focused on the recent advances of multiple miRNAs related to the progression of CADs, including As, AMI, HF, and ISR occurring after PCI. Additionally, the existent challenges and potentials of miRNAs serving as biomarkers are also evaluated, which aims to accelerate the applications of miRNAs in the clinics (Figure 2). As biomarkers, miRNAs have many advantages such as high specificity, high sensitivity, and convenient availability (Table 1), and show promising broad applications in the diagnosis, treatment as well as prognosis of the diseases. Although promising, several challenges still need to be addressed before they can be generally put into clinical applications.

Figure 2 miRNA biomarkers associated with the progression and prognosis of CADs. Atherosclerosis (As), acute myocardial infarction (AMI) and heart failure (HF) indicate three representative disease states during the progression of CAD, respectively. Besides, in-stent restenosis (ISR) is also shown to represent a common problem occurring after the PCI treatment. Here, representative miRNAs associated with these pathologies are listed in each frame.

Based on the advantages of miRNAs as hallmarks for various diseases, future work can be performed to integrate the detection of multiple diseases at one time by simultaneously examining multiple miRNAs. Additionally, further potentials other than biomarkers of miRNAs should also be exploited. Future researches should be directed to the development of miRNA-based drugs or miRNA-based scaffolds for the treatment and prevention of CADs. For example, several drug- and genemodified stents for preventing coronary restenosis have been developed, which provides promising strategy for the treatment as well as prevention of ISR by releasing miRNAs to modulate the mRNAs involved in the proliferation of $\mathrm{VSMCs}^{[64,}{ }^{66]}$. Therefore, particular attention should also be paid to optimize the stability, effectiveness of delivery as well as the sustained release of miRNA-based drugs. Moreover, to overcome current challenges and increase the efficacy of currently used miRNAs, great efforts remain to be made to gain more detailed mechanistic knowledge on miRNAs as well as their clear correlations with certain diseases.

\section{References}

1. Nowbar AN, Howard JP, Finegold JA, Asaria P, Francis DP. Int. J. Cardiol. 174: 293-8 (2014).

2. Fox KAA, Metra M, Morais J, Atar D. Nat Rev Cardiol 17: 9-21 (2020).

3. Al-Lamee RK, Nowbar AN, Francis DP. Heart 105: 11-9 (2019).

4. Her AY, Shin ES. Korean Circ J 48: 337-49 (2018).

5. Mirza AJ, Taha AY, Aldoori JS, Hawas JM, Hassan KW. Asian Cardiovasc Thorac Ann 26: 101-6 (2018).

6. Dai R, Liu Y, Zhou Y, Xiong X, Zhou W, et al. J Clin Lab Anal 34: e23013 (2020).

7. Li Z, Wu J, Wei W, Cai X, Yan J, et al. Front. Physiol. 
10(2019).

8. Zhou SS, Jin JP, Wang JQ, Zhang ZG, Freedman JH, et al. Acta Pharmacol Sin 39: 1073-84 (2018).

9. O'Brien J, Hayder H, Zayed Y, Peng C. Front. Endocrinol. 9(2018).

10. Mori MA, Ludwig RG, Garcia-Martin R, Brandao BB, Kahn CR. Cell Metab 30: 656-73 (2019).

11. Silva D, Carneiro FD, Almeida KC, FernandesSantos C. Arq Bras Cardiol 111: 738-46 (2018).

12. Bertoli G, Cava C, Castiglioni I. Theranostics 5: 1122-43 (2015).

13. Sayed AS, Xia K, Salma U, Yang T, Peng J. Heart Lung Circ 23: 503-10 (2014).

14. Sun YY, Qin SS, Cheng YH, Wang CY, Liu XJ, et al. Acta Pharmacol Sin 39: 885-92 (2018).

15. Sun LL, Li WD, Lei FR, Li XQ. J Cell Mol Med 22: 4568-87 (2018).

16. Wei X, Hou X, Li J, Liu Y. DNA and Cell Biology 36: 127-35 (2017).

17. Wei X, Hou X, Li J, Liu Y. DNA Cell Biol. 36: $127-$ 35 (2017).

18. Hajibabaie F, Kouhpayeh S, Mirian M, Rahimmanesh I, Boshtam M, et al. J Physiol Biochem (2019).

19. Huang B, Lv H, Song Y. Sensors (Basel) 19(2018).

20. Huang HT, Wang F, Ding SG, Tian YB, Xu YM, Zhong CJ. Acta Medica Mediterranea 35: 109-16 (2019).

21. Lin J, He S, Sun X, Franck G, Deng Y, et al. FASEB J 30: 3216-26 (2016).

22. Ørom UA, Nielsen FC, Lund AH. Mol. Cell 30: 46071 (2008).

23. Vasudevan S, Tong Y, Steitz JA. Science 318: 19314 (2007).

24. Zhang J, Xing Q, Zhou X, Li J, Li Y, et al. Mol Med Rep 16: 7766-74 (2017).

25. Liu G, Niu X, Meng X, Zhang Z. J Thorac Dis 10: 3206-15 (2018).

26. Cheng M, An S, Li J. Medicine (Baltimore) 96: e7741 (2017).

27. Kataoka M, Wang DZ. Cells 3: 883-98 (2014).

28. Quintavalle M, Condorelli G, Elia L. Vasc. Pharmacol. 55: 106-10 (2011).

29. Joppich M, Weber C, Zimmer R. Thromb Haemost 119: 1247-64 (2019).

30. Tibaut M, Caprnda M, Kubatka P, Sinkovic A, Valentova V, et al. Heart Lung Circ 28: 667-77 (2019).

31. Lv JX, Kong Q, Ma X. Chronic Dis Transl Med 3: 207-12 (2017).

32. Bruen R, Fitzsimons S, Belton O. Front Pharmacol 10: 463 (2019).

33. Li X, Kong D, Chen H, Liu S, Hu H, et al. Sci Rep 6: 21789 (2016).

34. McDonald RA, Halliday CA, Miller AM, Diver LA, Dakin RS, et al. J Am Coll Cardiol 65: 2314-27
(2015).

35. Mythili S, Malathi N. Biomed Rep 3: 743-8 (2015).

36. Wang F, Long G, Zhao C, Li H, Chaugai S, et al. PLoS One 9: e105734 (2014).

37. Xue S, Zhu W, Liu D, Su Z, Zhang L, et al. Mol Med 25: 18 (2019).

38. Horie T, Baba O, Kuwabara Y, Chujo Y, Watanabe S, et al. J Am Heart Assoc 1: e003376 (2012).

39. Savic-Radojevic A, Pljesa-Ercegovac M, Matic M, Simic D, Radovanovic S, Simic T. pp. 93-152(2017).

40. Zhang R, Niu H, Ban T, Xu L, Li Y, et al. Int J Cardiol 166: 259-60 (2013).

41. Florio MC, Magenta A, Beji S, Lakatta EG, Capogrossi MC. Curr Probl Cardiol: 100406 (2018).

42. Masson S, Batkai S, Beermann J, Bar C, Pfanne A, et al. Eur J Heart Fail 20: 78-85 (2018).

43. Gabbasov Z, Kozlov S, Melnikov I, Byazrova S, Saburova O, et al. Clin. Appl. Thromb.-Hemost. 24: 1308-14 (2018).

44. Claessen BE, Stone GW, Mehran R, Witzenbichler B, Brodie BR, et al. J. Thromb. Thrombolysis 34: 165-79 (2012).

45. Yu Z-H, Wang H-T, Tu C. Eur. J. Med. Res. 22(2017).

46. O'Sullivan JF, Neylon A, Fahy EF, Yang P, McGorrian C, Blake GJ. Heart Asia 11: e011134 (2019).

47. Long G, Wang F, Duan Q, Yang S, Chen F, et al. PLoS One 7: e50926 (2012).

48. Devaux Y, Vausort M, McCann GP, Kelly D, Collignon O, et al. PLoS One 8: e70644 (2013).

49. He X, Ji J, Wang T, Wang MB, Chen XL. Cardiology 138: 107-14 (2017).

50. Wang H, Eitzman DT. Atherosclerosis 229: 18-22 (2013).

51. Wang Y, Chang W, Zhang Y, Zhang L, Ding H, et al. J. Cell. Physiol. 234: 4778-86 (2019).

52. De Rosa S, Fichtlscherer S, Lehmann R, Assmus B, Dimmeler S, Zeiher AM. Circulation 124: 1936-44 (2011).

53. Cheng Y, Wang X, Yang J, Duan X, Yao Y, et al. J Mol Cell Cardiol 53: 668-76 (2012).

54. Grabmaier U, Clauss S, Gross L, Klier I, Franz WM, et al. Int J Cardiol 244: 30-6 (2017).

55. Liu X, Fan Z, Zhao T, Cao W, Zhang L, et al. Exp Gerontol 72: 230-8 (2015).

56. Wang GK, Zhu JQ, Zhang JT, Li Q, Li Y, et al. Eur. Heart J. 31: 659-66 (2010).

57. Boon RA, Dimmeler S. Nat Rev Cardiol 12: 135-42 (2015).

58. Zeller T, Keller T, Ojeda F, Reichlin T, Twerenbold R, et al. Eur Heart J 35: 2106-14 (2014).

59. van Almen GC, Verhesen $\mathrm{W}$, van Leeuwen RE, van de Vrie M, Eurlings C, et al. Aging Cell 10: 769-79 (2011).

60. Cruz MS, da Silva AMG, de Souza KSC, Luchessi 
AD, Silbiger VN. Heart Fail Rev 25: 321-9 (2020).

61. Wong LL, Rademaker MT, Saw EL, Lew KS, Ellmers LJ, et al. Sci Rep 7: 8250 (2017).

62. Schaer GL, Zhang C. J Am Coll Cardiol 65: 232830 (2015).

63. Andreini D, Mushtaq S, Pontone G, Conte E, Collet C, et al. JACC Cardiovasc Imaging 13: 732-42 (2020).

64. Lekshmi KM, Che H-L, Cho C-S, Park I-K. Chonnam Med J 53(2017).

65. Che HL, Bae IH, Lim KS, Uthaman S, Song IT, et al. Korean Circ. J. 46: 23-32 (2016).
66. Izuhara M, Kuwabara Y, Saito N, Yamamoto E, Hakuno D, et al. PLoS One 12: e0172798 (2017).

67. Dong S, Cheng Y, Yang J, Li J, Liu X, et al. J Biol Chem 284: 29514-25 (2009).

68. Zhou X, Mao A, Wang X, Duan X, Yao Y, Zhang C. PLoS One 8: e62245 (2013).

69. Ban E, Chae DK, Yoo YS, Song EJ. Anal Bioanal Chem 409: 6397-404 (2017).

70. Ye J, Xu M, Tian X, Cai S, Zeng S. J Pharm Anal 9: 217-26 (2019). 